\title{
Cinnamaldehyde Exerts Its Antifungal Activity by Disrupting the Cell Wall Integrity of Geotrichum citri-aurantii
}

\author{
Qiuli OuYang, Xiaofang Duan, Lu Li and Nengguo Tao* \\ School of Chemical Engineering, Xiangtan University, Xiangtan, China
}

Our previous study showed that cinnamaldehyde (CA) significantly inhibited the mycelial growth of Geotrichum citri-aurantii, one of the main postharvest pathogens in citrus fruits. This study investigated the antifungal mechanism of CA against G. citri-aurantii. Scanning electron microscopy (SEM) and transmission electron microscopy (TEM) images showed that CA treatment led to clear morphological changes in the cell walls and membranes of G. citri-aurantii. However, the membrane integrity, total lipids and ergosterol contents were not apparently affected by CA treatment. Notably, the extracellular alkaline phosphatase (AKP) activity was increased after CA treatment, suggesting impairment in cell wall permeability. A weakened fluorescence in the cell wall, a decrease in the chitin contents, and changes of ten genes involved in cell wall

OPEN ACCESS

Edited by:

Juan Aguirre,

Universidad de Chile, Chile

Reviewed by: Jose Luis Henriquez, Universidad de Chile, Chile

Jorge Reinheimer,

National University of the Littoral,

Argentina

${ }^{*}$ Correspondence:

Nengguo Tao

nengguotao@126.com

Specialty section:

This article was submitted to

Food Microbiology,

a section of the journal

Frontiers in Microbiology

Received: 19 July 2018

Accepted: 14 January 2019

Published: 30 January 2019

Citation:

OuYang Q, Duan X, LiL and Tao N (2019) Cinnamaldehyde Exerts Its Antifungal Activity by Disrupting the Cell Wall Integrity of Geotrichum citri-aurantii. Front. Microbiol. 10:55

doi: 10.3389/fmicb.2019.00055 integrity were also observed. These results suggested that CA may exhibit its antifungal activity against $G$. citri-aurantii by interfering the build of cell wall and therefore lead to the damage of cell wall permeability and integrity.

Keywords: Geotrichum citri-aurantii, Cinnamaldehyde, cell membrane, cell wall, antifungal mechanism

\section{INTRODUCTION}

Worldwide citrus sour rot caused by Geotrichum citri-aurantii, which is less common than green and blue molds, has been reported as an important postharvest disease of citrus fruits, particularly during periods of high rainfall (McKay et al., 2012; Zhou et al., 2014). Currently, sour rot cannot be actively controlled by the registered fungicides imazalil, thiabendazole, pyrimethanil and fludioxonil and can only be partially controlled with the commercial fungicides sodium $o$-phenylphenate (SOPP) and propiconazole (McKay et al., 2012). However, the concentration of SOPP required to effectively manage sour rot can result in the development of oleocellosis and darkening of the fruit rind (Regnier et al., 2014; Zhou et al., 2014). Therefore, the use of essential oils represents a promising strategy for the management of citrus sour rot due to their effective and commercial characteristics.

Many studies highlight the feasibility of applying essential oils for postharvest disease control in citrus due to their notable antifungal activity against G. citri-aurantii and substantially lower risk for the development of resistance (Talibi et al., 2012; Regnier et al., 2014; Wuryatmo et al., 2014). Cinnamaldehyde (CA), a major constituent of cinnamon essential oils, was demonstrated to have potent antifungal activities against a wide variety of fungi (Bang et al., 2000; Wang et al., 2005; Wu et al., 2017; Duan et al., 2018). Our previous study showed that CA significantly inhibited the mycelial growth of G. citri-aurantii with a minimum inhibitory concentration (MIC) and minimum fungicidal concentration (MFC) of 
$0.50 \mathrm{~mL} / \mathrm{L}$ (Wu et al., 2017). Furthermore, wax + CA treatments had strong inhibition effects on sour rot and green mold decay during storage time and induced significant citrus fruit defense responses against the pathogens (Wu et al., 2017; Duan et al., 2018). Therefore, CA may be a potential candidate as an effective and environmentally benign citrus preservative.

Until now, the antifungal mechanism of CA was widely investigated but there were no conclusive findings. Previous studies reported that CA acts by perturbing the cell membrane, acting as an ATPase inhibitor, inhibiting enzymes involved in cytokine interaction, or inhibiting cell wall biosynthesis (Smid et al., 1996; Bang et al., 2000; Xie et al., 2004; Shreaz et al., 2011, 2013; Xing et al., 2014). In a study on Saccharomyces cerevisiae, trans-cinnamaldehyde caused a partial collapse of the integrity of the cytoplasmic membrane, leading to the excessive leakage of metabolites and enzymes of the cell and finally loss of viability (Smid et al., 1996). However, Bang et al. (2000) found that CA inhibited the growth of S. cerevisiae cells because of its ability to inhibit cell wall-synthesizing enzymes, $\beta$-(1,3)-glucan synthase and chitin synthase I. In another study, CA inhibited the growth of Phytophthora capsici by disturbing calcium homeostasis (Hu et al., 2013). Xie et al. (2004) and Xing et al. (2014) demonstrated that CA significantly inhibited the growth of Aspergillus flavus and Fusarium verticillioides by causing irreversible deleterious morphological and ultrastructural alterations, such as the lack of cytoplasmic contents, loss of integrity and rigidity of the cell wall, disruption of the plasma membrane, and destruction of mitochondria. Recently, Shreaz et al. (2011) demonstrated that CA exerted its antifungal activity by targeting the sterol biosynthesis of Candida, whereas in another study, the immediate effect of CA against Candida may originate from the inhibition of PM-ATPase and a decrease of $\mathrm{pHi}$, or may contribute to the depletion of NADPH (Shreaz et al., 2013). Nevertheless, to our knowledge, there are no reports on the underlying mechanism of the antifungal action of CA against G. citri-aurantii. Thus, the inhibitory mechanism of CA against $G$. citri-aurantii requires further studies.

The objective of this research was to reveal the antifungal mechanism of CA against $G$. citri-aurantii by determining the following: (i) the morphology and ultrastructure of cell membranes using SEM and TEM, (ii) the plasma membrane integrity and the total lipid and ergosterol contents, (iii) the extracellular alkaline phosphatase (AKP) activity, the calcofluor white stain results and chitin contents, (iv) and the expression levels of genes involved in cell walls.

\section{MATERIALS AND METHODS}

\section{Fungal Strain}

Geotrichum citri-aurantii was provided by the Department of Biotechnology and Food Engineering, Xiangtan University, Xiangtan, China. The fungal pathogen G. citri-aurantii used in this study was isolated from infected citrus fruit (Zhou et al., 2014) and cultured on potato dextrose agar (PDA) at $28 \pm 2^{\circ} \mathrm{C}$.
A spore suspension $\left(5 \times 10^{5}\right.$ spores $\left./ \mathrm{mL}\right)$ in potato dextrose broth (PDB) was prepared using a hemocytometer.

\section{Preparation of CA Treatments}

For solid culture, CA (Sigma, St. Louis, MO, United States) was added into PDA (with $0.05 \%$ Tween-80) for a final concentration of $0.25 \mu \mathrm{L} / \mathrm{mL}$ (1/2MIC). A $6 \mathrm{~mm}$ inoculum disk cut with a cork borer from the leading edge of the fungal culture on PDA plates was placed at the center of each new plate. Culture plates were then incubated at $28 \pm 2^{\circ} \mathrm{C}$ for 4 days.

For liquid culture, CA was added into $\mathrm{PDB}$ for a final concentration of $0.25 \mu \mathrm{L} / \mathrm{mL}$ (1/2MIC). Two hundred milliliters spore suspension $\left(5 \times 10^{5}\right.$ spores $\left./ \mathrm{mL}\right)$ were added into $40 \mathrm{~mL}$ PDB containing CA and incubated in a moist chamber with 160 $\mathrm{r} / \mathrm{min}$ at $28 \pm 2{ }^{\circ} \mathrm{C}$ for $0,30,60$, and $120 \mathrm{~min}$.

\section{Scanning Electron Microscopy (SEM)}

The 4-day-old fungal culture described above was directly examined with a JEOL JSM-6360LV SEM (JEOL, Tokyo, Japan). The hyphae grown on PDA without CA were used as a control. The procedures for the SEM observation were described in our previous study (Zhou et al., 2014).

\section{Transmission Electron Microscopy (TEM)}

The 4-day-old fungal culture described above was directly examined with a transmission electron microscope (JEM-1230; JEOL Ltd., Tokyo, Japan) operated at an accelerating voltage of $80 \mathrm{kV}$. The hyphae grown on PDA without CA were used as a control. The procedures for the TEM observation were described in our previous study (Tao et al., 2014).

\section{Assay for Plasma Membrane Integrity}

Plasma membrane integrity of the G. citri-aurantii with different CA treatments in PDB described above was analyzed by propidium iodide (PI) staining coupled with an F97 PRO fluorescence spectrophotometer (Lengguang Technology, Shanghai, China) (OuYang et al., 2018).

\section{Determination of Ergosterol Content}

Total ergosterol contents of G. citri-aurantii cells with different CA treatments in PDB described above were determined using the HPLC method (OuYang et al., 2016). The fungal culture on PDA without CA was used as a control. The samples were dried with a vacuum freeze drier for $4 \mathrm{~h}$. About $0.1 \mathrm{~g}$ of dry mycelia were homogenized with liquid nitrogen and saponified by adding $4 \mathrm{~mL}$ of freshly prepared $30 \%$ (w/v) methanolic $\mathrm{KOH}$ and $8 \mathrm{~mL}$ of absolute ethanol at $90{ }^{\circ} \mathrm{C}$ for $2 \mathrm{~h}$. The mixtures were extracted with $3 \mathrm{~mL}$ petroleum ether for three times and washed by saturated $\mathrm{NaCl}$ solution twice. The samples were then vacuum concentrated and dissolved in $10 \mathrm{~mL}$ absolute ethanol.

HPLC was conducted on a Shimadzu LC-20AT liquid chromatography system (Shimadzu Scientific Instrument, Japan) equipped with a model LC-20AT solvent delivery system, a model SPD-M20A photo diode array detection system, and an Empower Chromatography Manager. The sample extracts were separated and analyzed by using a C18 column $(250 \mathrm{~mm} \times 4.6 \mathrm{~mm}, 5 \mathrm{~mm})$ 
at room temperature. The mobile phase consisted of solvent $\mathrm{A}$ (methanol). The flow rate was $1.0 \mathrm{~mL} / \mathrm{min}$. Chromatographic peaks were identified by comparing the retention times and spectra against the known standard. The detecting wavelength was set at $282 \mathrm{~nm}$. Aliquots of $20 \mathrm{~mL}$ were directly injected into the HPLC for the determination. All injections were repeated three times.

\section{Determination of Total Lipid Content}

Total lipid contents of G. citri-aurantii cells with different CA treatments in PDB described above were determined using the phosphovanillin method (Tao et al., 2014). The fungal culture in PDB without CA was used as a control. The samples were dried with a vacuum freeze drier for $4 \mathrm{~h}$. About $0.1 \mathrm{~g}$ of dry mycelia were homogenized with liquid nitrogen and extracted with $4.0 \mathrm{~mL}$ of methanol/chloroform/water mixture $(2: 1: 0.8, \mathrm{v} / \mathrm{v} / \mathrm{v})$ in a clean dry test tube with vigorous shaking for $30 \mathrm{~min}$. The tubes were centrifuged at $4000 \mathrm{~g}$ for $10 \mathrm{~min}$. The lower phase containing lipids was thoroughly mixed with $0.2 \mathrm{~mL}$ saline solution and centrifuged at $4000 \mathrm{~g}$ for $10 \mathrm{~min}$. Then, an aliquot of $0.2 \mathrm{~mL}$ chloroform and lipid mixture was transferred to a novel tube and $0.5 \mathrm{~mL} \mathrm{H}_{2} \mathrm{SO}_{4}$ was added, heated for $10 \mathrm{~min}$ in a boiling water bath. After that, $3 \mathrm{~mL}$ phosphovanillin was added and shake vigorously, and then incubated at room temperature for $10 \mathrm{~min}$. The absorbance at $520 \mathrm{~nm}$ was utilized to calculate total lipid contents from the standard calibration curve using cholesterol as a standard.

\section{Assays for Extracellular Alkaline Phosphatase (AKP) Activity}

The extracellular AKP activities of G. citri-aurantii mycelia with different CA treatments in PDB described above were assayed by a UV-2450 UV/Vis spectrophotometer [Shimadzu (China) Co., Ltd., Shanghai, China] using the AKP kit (Solarbio Science and Technology Co., Ltd., Beijing, China) following the instructions. The fungal culture in PDB without CA was used as a control. Each experiment was repeated three times. The enzyme activity was expressed as $\mathrm{U} / \mathrm{g}$ prot.

\section{The Effect of CA on the Cell Wall Integrity of G. citri-aurantii}

The effects of CA on the cell wall integrity of G. citri-aurantii were analyzed by calcofluor white (Sigma, St. Louis, MO, United States) staining coupled with fluorescence microscopy. The 2-day-old mycelia were collected from $50 \mathrm{~mL}$ PDB containing 1/2MIC CA and centrifuged at $4000 \mathrm{~g}$ for $10 \mathrm{~min}$. The collected mycelia were stained with $10 \mu \mathrm{L}$ of calcofluor white stain after the addition of $10 \mu \mathrm{L} \mathrm{KOH} \mathrm{(10 \% )} \mathrm{following}$ the manufacturer's instructions. Samples were observed with a fluorescence microscope (Nikon ECLIPSE TS100, Japan). The fungal culture in $\mathrm{PDB}$ without $\mathrm{CA}$ was used as a control.

\section{Determination of the Chitin Contents}

The chitin contents of $G$. citri-aurantii cells with different CA treatments in PDB described above were determined by the following steps. The samples were dried and ground into a powder. Then, the powders $\left(\mathrm{W}_{1}\right)$ were treated with a saturated $\mathrm{KOH}$ solution at $160^{\circ} \mathrm{C}$ for at least 15 min until the products became a transparent thin film. Then, the products were poured onto filter paper and rinsed slowly with distilled water. The extracts were successively dehydrated by 95 and 100\% alcohol, weighing again as $\mathrm{W}_{2}$. The chitin contents were calculated by the following formula (Jiang, 2015):

$$
\text { chitin content }(\%)=\mathrm{W}_{2} / \mathrm{W}_{1} \times 1.26 \times 100 \%
$$

where 1.26 is the conversion factor.

\section{Real-Time Fluorescence Quantitative PCR (RTFQ-PCR) Analysis}

The G. citri-aurantii cells treated for 0,30 , and $60 \mathrm{~min}$ were collected from PDB containing 1/2MIC of CA. RNA was extracted from G. citri-aurantii cells using the Trizol reagent (Invitrogen, United States) following the manufacturer's instructions. Two micrograms of DNAfree RNA were used for reverse transcription by M-MLV (Promega, United States) with oligo dT18. RTFQ-PCR was performed on a BIO-RAD CFX Connect Thermal Cycler (Bio-Rad, CA, United States) using the FastStart Universal SYBR Green Master (Roche, Switzerland). The primers for the tested genes were designed base on our RNA-Seq data of G. citri-aurantii (data not shown) and listed in Table 1. RTFQ-PCR was programmed as follows: $95^{\circ} \mathrm{C}$ for $10 \mathrm{~min}$ followed by 40 cycles of $95^{\circ} \mathrm{C}$ for $15 \mathrm{~s}$ and $60^{\circ} \mathrm{C}$ for

TABLE 1 | Primer pair sequences designed for validation of differentially expressed genes in control and 1/2MIC CA treatment of G. citri-aurantii using Real-time Fluorescence Quantitative PCR (RTFQ-PCR).

\begin{tabular}{|c|c|}
\hline Gene name & Primer sequence $\left(5^{\prime}-3^{\prime}\right.$, forward/reverse $)$ \\
\hline \multirow[t]{2}{*}{ r-actin } & TTACGCCGGTTTCTCCCTCC \\
\hline & GACGATTTCACGCTCGGCAG \\
\hline \multirow[t]{2}{*}{ CHS5 } & GCGCCAAACCGCTACAAGAC \\
\hline & TGATCCATTCAGGGCGCACG \\
\hline \multirow[t]{2}{*}{ CHS2 } & TACCAGCTTCTCCGGGCTTG \\
\hline & GCAACACACCACAGGACGAC \\
\hline \multirow[t]{2}{*}{ chs A } & CCGCCTATTCCCGATACCGC \\
\hline & CCGCAAGAACAGCCACAGAC \\
\hline \multirow[t]{2}{*}{$c h s B$} & GTTCTCATGGCGCTTGGCTC \\
\hline & GTGCCAGTTGCAGAAAGCGT \\
\hline \multirow[t]{2}{*}{ chsG } & GCTGGGAAATCGGCCAAAGG \\
\hline & CGGACCGCCAGGTAAAATGC \\
\hline \multirow[t]{2}{*}{$\mathrm{CH} 1$} & CGCGTGCCAAAGCTGACTAC \\
\hline & GCGCCGACAAGGGATTTCTG \\
\hline \multirow[t]{2}{*}{ ChiA1 } & CGTTGTTCCCAAGCCAACCC \\
\hline & CACAGAAGCGCTGGCAGAGT \\
\hline \multirow[t]{2}{*}{ UAP1 } & ATGTGGACCTAACCACGGGC \\
\hline & ACGATACACCTCCAGCGAGC \\
\hline \multirow[t]{2}{*}{ Glu1 } & TTATCCATGCGCTCCCCCAC \\
\hline & CGCTGATAGAGTCACCGCCA \\
\hline \multirow[t]{2}{*}{ Glu2 } & TACTGGATGGTCAACGGCGG \\
\hline & TGTGGTGCCAGAGTGGTCAG \\
\hline
\end{tabular}


$1 \mathrm{~min}$. The $2^{-\Delta \Delta \mathrm{CT}}$ method was used to quantify the value of each sample using the $r$-actin gene as an internal reference (Livak and Schmittgen, 2001).

\section{Statistical Analysis}

All data were expressed as the mean \pm standard deviation of three independent replicates. A one-way analysis of variance (ANOVA) followed by Duncan's test was performed to test the significance of differences between means obtained among the treatments at the 5\% level of significance using the SPSS statistical software package release 16.0 (SPSS Inc., Chicago, IL, United States).

\section{RESULTS}

\section{Morphology Change of CA Treated G. citri-aurantii by SEM}

Analysis of SEM images showed that the control samples exhibited normal morphology with regular, homogenous and robust hyphae of a constant diameter and a smooth surface (Figure 1A). However, the mycelia after CA treatment showed considerable alterations, which appeared as a damaged surface with rips and partly squashed, distorted, and shriveled mycelia (Figure 1B).

\section{Cell Wall Damage of CA Treated G. citri-aurantii by TEM}

Analysis of TEM images showed that the untreated healthy mycelia showed a cell wall with uniform layers, defined plasma-membrane and periplasm region with normal thickness. The cytoplasm was clear, and well-organized mitochondria were scattered throughout the cytoplasm (Figure 1C). By contrast, CA damaged the cell wall and cell membrane of G. citri-aurantii. The hyphal wall was disrupted and even disappeared in some regions. The plasmalemma appeared faint, undefined and irregular. Discrete cytoplasmic alterations, including a very dense cytoplasm and a mass of disorganized structures, were also observed (Figure 1D).

\section{Effect of CA on the Plasma Membrane Integrity of G. citri-aurantii}

According to the PI staining results, the hyphae after $120 \mathrm{~min}$ of CA treatment presented a high fluorescence intensity (Figure 2, $P<0.05$ ), which was generated by the damage to the plasma membrane integrity. However, the fluorescence intensity in CA treated samples before $60 \mathrm{~min}$ of exposure were lower than in the control, suggesting that the plasma membrane integrity was not damaged during this period.

\section{Effect of CA on the Total Lipid Content of G. citri-aurantii}

The effect of CA on the total lipid content of G. citri-aurantii cells is shown in Figure 3A. The total lipid contents in control samples remained stable during the incubation time, whereas the CA treatment significantly induced the accumulation of total lipid contents at $60 \mathrm{~min}$ of exposure. The total lipid content of the CA treatment at $60 \mathrm{~min}$ was $346.0 \pm 20.1 \mathrm{mg} / \mathrm{g} \mathrm{DW}$, which was significantly higher than the control $(213.1 \pm 15.5 \mathrm{mg} / \mathrm{g} \mathrm{DW})$ $(P<0.05)$. Nevertheless, the total lipid contents of CA treatment
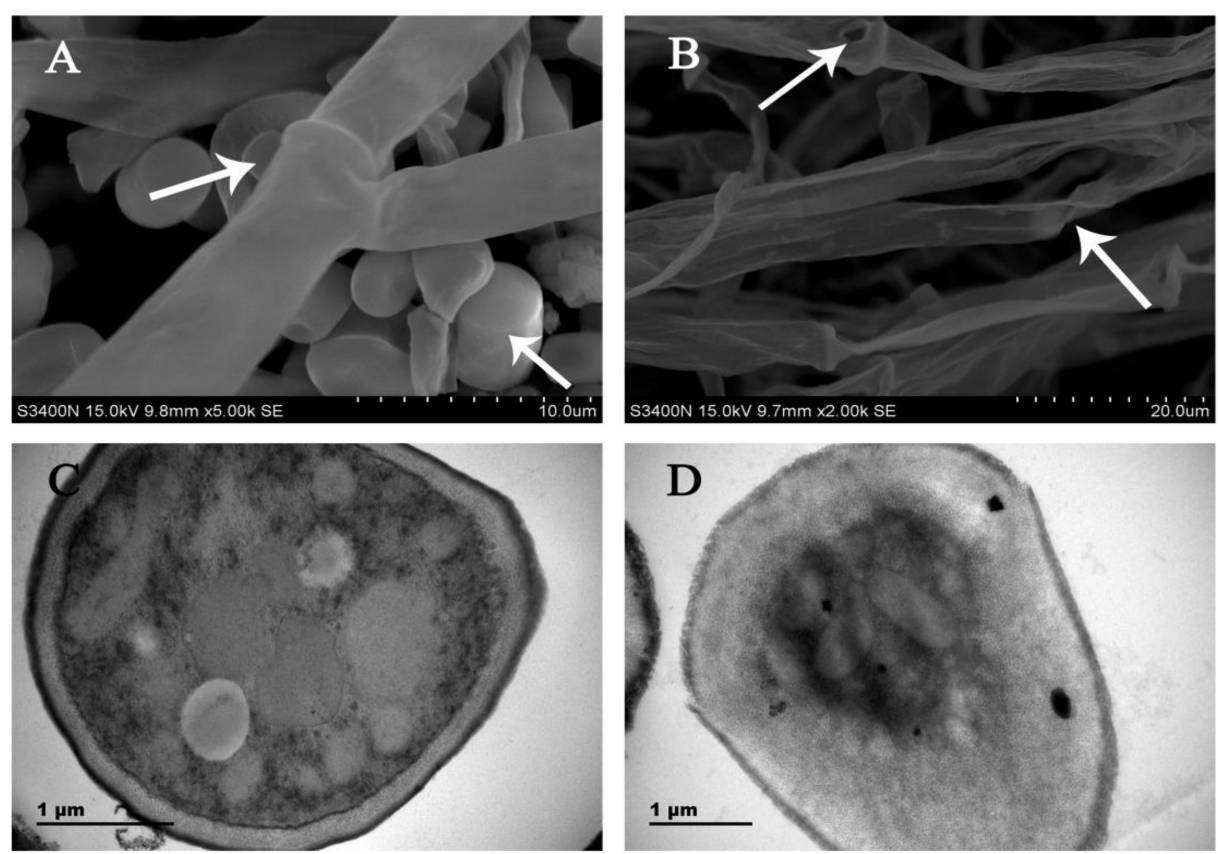

FIGURE 1 | SEM images of (A) untreated control culture of G. citri-aurantii for 4 days; (B) culture after incubation with the CA for 4 days. TEM images of (C) untreated control culture of G. citri-aurantii for 4 days; (D) culture after incubation with the CA for 4 days. 


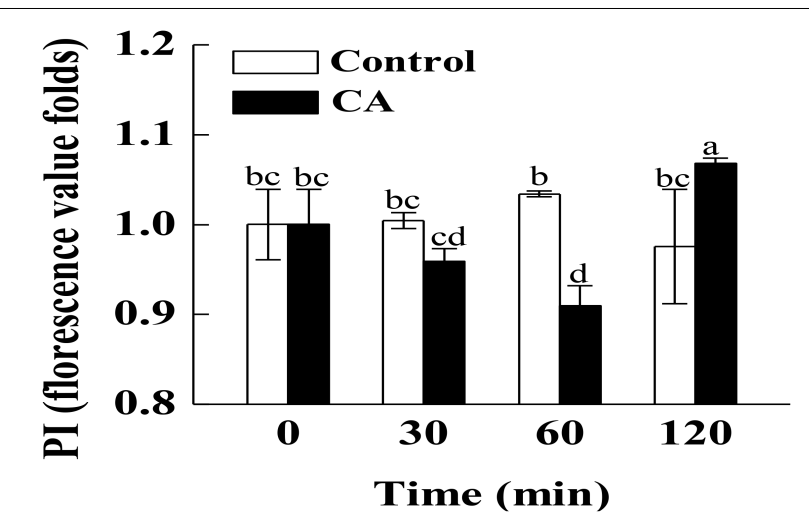

FIGURE 2 | Effects of CA on the plasma membrane integrity of G. citri-aurantii mycelia. The data presented are the means of pooled data. Error bars indicate the SDs of the means $(n=3)$. Bars with different lower-case letters between different groups indicate significant differences according to duncan's test $(P<0.05)$.

decreased after 60 min of exposure and no difference in the total lipid contents were observed between the 120 min treatment and the control.

\section{Effect of CA on the Ergosterol Content of G. citri-aurantii}

There was no significant difference in the ergosterol contents of the CA treatments and control during the entire period. At $120 \mathrm{~min}$ of exposure, the ergosterol contents in the treated and control groups were $5.01 \pm 0.15$ and $4.69 \pm 0.42 \mathrm{mg} / \mathrm{g} \mathrm{DW}$, respectively (Figure 3B).

\section{Effect of CA on the Extracellular AKP Activity of G. citri-aurantii}

At 30 min of exposure, the extracellular AKP activity in the CA treatment was $5.06 \pm 0.18 \mathrm{U} / \mathrm{g}$ prot, which was significantly higher than the control $(4.52 \pm 0.16 \mathrm{U} / \mathrm{g}$ prot). This change became more evident with increasing exposure time $(P<0.05)$. At $120 \mathrm{~min}$ of exposure, the extracellular AKP activity in the CA treatment was $6.18 \pm 0.09 \mathrm{U} / \mathrm{g}$ prot, which was significantly higher than the control $(4.42 \pm 0.03 \mathrm{U} / g$ prot $)$ (Figure 4A).

\section{Effect of CA on the Cell Wall of G. citri-aurantii}

As shown in Figure 4B, the fluorescence of the control cells was uniformly distributed, and the septa were visible with brighter fluorescent lines because septa are particularly rich in chitin (Figures 4B1-B3). After exposure to CA, the fluorescence in the cell walls was uneven and the fluorescence in the septa became weaker (Figures 4B4-B6).

\section{Effect of CA on the Chitin Contents of G. citri-aurantii}

The chitin contents of $G$. citri-aurantii mycelia rapidly decreased $(P<0.05)$ with the CA treatment before $60 \mathrm{~min}$ of exposure. After $120 \mathrm{~min}$ of exposure, the chitin content in the CA treated samples was $8.25 \pm 0.36 \%$, which was significantly lower than the control samples $(12.06 \pm 0.80 \%)(P<0.05)$ (Figure 4C).

\section{Effects of CA on the Gene Expressions Involved in the Cell Wall Integrity}

After $30 \mathrm{~min}$ of exposure to $\mathrm{CA}$, the expression levels of CHS2, chs A, chsB, chsG, and UAP1 involved in chitin biosynthesis were significantly lower than the control samples. By contrast, the expression level of CHI1 related to chitin decomposition in CA treatments was significantly higher than the control sample at $30 \mathrm{~min}$ of incubation. Moreover, the expression levels of $\operatorname{chs} A, \operatorname{chs} B$ and UAP1 in CA treated samples were equal to those in control samples at $60 \mathrm{~min}$ of incubation, whereas the gene expression of CHS2, CHS5 and chs $G$ were all repressed by CA treatment at this time. The expression level of another gene CHIA1 involved in chitin decomposition had no difference between CA treatments and the control samples. The gene expression levels of endo-1,3(4)- $\beta$-glucanase (Glu1) and glucan 1,3- $\beta$-glucosidase (Glu2), associated with glucans hydrolysis, were significantly
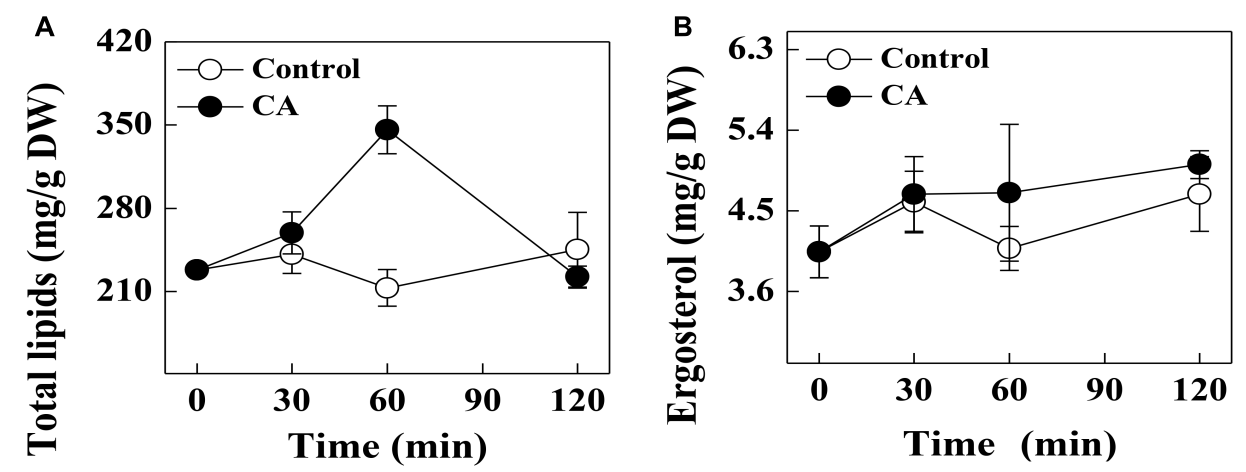

FIGURE 3 | Effects of CA on (A) the total lipids contents and (B) ergosterol contents of $\mathbf{G}$. citri-aurantii mycelia. The data presented are the means of pooled data. Error bars indicate the SDs of the means $(n=3)$. 


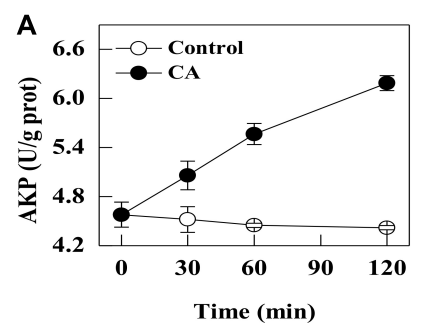

B
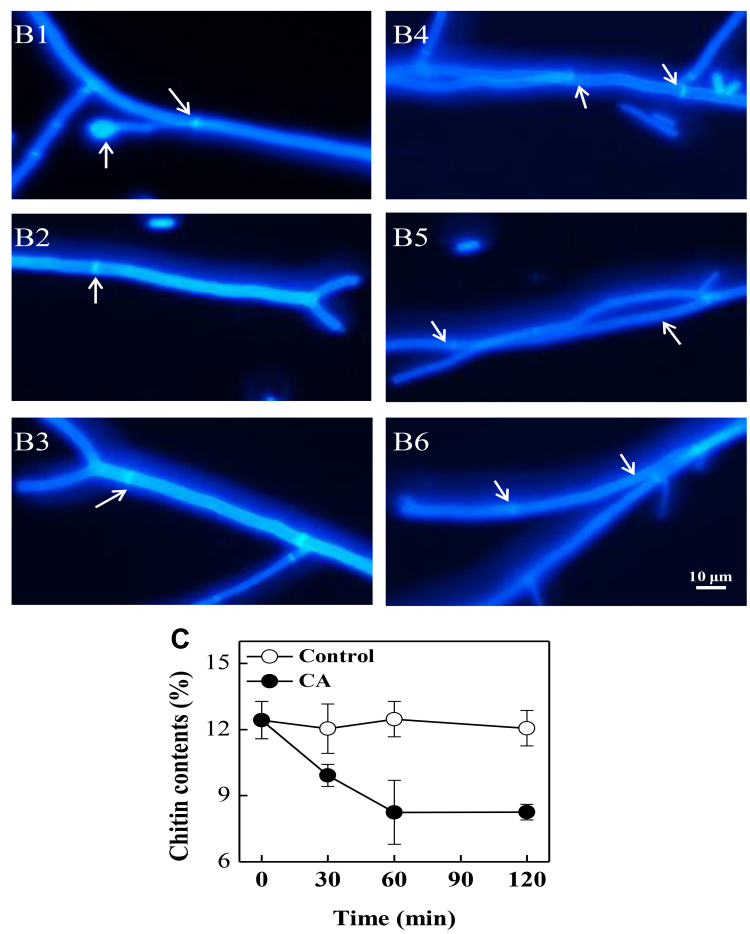

FIGURE 4 | The effects of CA on the cell walls of G. citri-aurantii. (A) The extracellular AKP activity of G. citri-aurantii; (B) G. citri-aurantii treated with the $1 / 2 \mathrm{MIC}$ CA observed under a fluorescence microscopy after staining with calcofluor white stain ( $\times 100$ magnification): (B1-B3) control group treated for 30, 60, and $120 \mathrm{~min}$, respectively; (B4-B6) 1/2MIC CA group treated for 30, 60 , and 120 min, respectively. (C) The chitin content of G. citri-aurantii. The data presented are the means of pooled data. Error bars indicate the SDs of the means $(n=3)$.

lower than the control groups at 30 and $60 \mathrm{~min}$ of exposure, respectively (Figure 5).

\section{DISCUSSION}

The lack of registered fungicides in controlling G. citri-aurantii infections in citrus fruit promotes the need to explore new sources of antifungal substances. Essential oils are promising alternatives to conventional fungicides for controlling postharvest diseases (Talibi et al., 2012; Shao et al., 2013, 2015; Regnier et al., 2014; Wei et al., 2018). Previously, CA was demonstrated to be effective in controlling some postharvest pathogens, including $P$. digitatum and G. citri-aurantii, and decrease the disease index of sour rot and green mold decay of citrus (Wu et al., 2017; Duan et al., 2018). This study aims to explore the possible antifungal mechanism of CA against the mycelial growth of $G$. citri-aurantii. In our study, CA caused distorted, shriveled, and squashed hyphae, and structural disorganization of the cytoplasm, which was consistent with the previous literature that essential oils could elicit versatile roles on cell walls, cell membranes, and cytoplasmic contents (Xie et al., 2004; Tao et al., 2014; Xing et al., 2014; Zhou et al., 2014; Shao et al., 2015; Wei et al., 2018).

The integrity of the plasma membrane played a crucial role in maintaining fungal viability (Shao et al., 2013; Tao et al., 2014). Earlier studies demonstrated that essential oils could increase the cell membrane permeability and damage the membrane integrity by decreasing the important cell membrane structure components, total lipids and ergosterol (Shreaz et al., 2011; Tao et al., 2014; OuYang et al., 2016). Shreaz et al. (2011) reported that $\mathrm{CA}$ exerted its antifungal activity by targeting the sterol biosynthesis of Candida. However, in our study, the addition of CA did not decrease the contents of total lipids and ergosterol of G. citri-aurantii. Interestingly, CA induced the massive accumulation of total lipids before $60 \mathrm{~min}$ of exposure (Figure 3A). This might lead to the thickening of cell membranes and reduce the amount of PI that enters cell membrane and thus decreased the fluorescence intensity. After $120 \mathrm{~min}$ of exposure, the total lipid content in CA treated group was rapidly decreased but remained at a similar level to that of control group. Correspondingly, the PI stain experiment results showed that the membrane integrity was significantly disrupted. These results suggested that the antifungal activity of CA is likely not caused by damage to the membrane permeability and integrity. This discrepancy may be caused by a difference in the structure of different fungal species. For example, citral was reported to inhibit the ergosterol biosynthesis of $P$. italicum and $P$. digitatum (Tao et al., 2014; OuYang et al., 2016), whereas the ergosterol content of Candida albicans was not influenced by citral (Leite et al., 2014).

The fungal wall is a flexible structure that provides protection to the cell and therefore is crucial for fungal permeability (Klis, 1994). The cell wall structure of fungi may be severely affected by essential oils including Hyssopus officinalis oil and tea tree oil (Ghfir et al., 1997; Shao et al., 2013). AKP is an enzyme produced in the cytoplasm and leaked into the periplasmic space. Generally, AKP releases from fungal cells with impaired cell wall permeability (Yang et al., 2016). In a previous study, tea tree oil was reported to cause the leakage of AKP and thus damaged the cell wall integrity of Botrytis cinerea (Shao et al., 2013). In this study, a significantly higher AKP activity was also observed in CA treatments, indicating that CA disrupted the cell wall permeability of $G$. citri-aurantii. Chitin, a $\beta$ $(1,4)$-linked polymer of $\mathrm{N}$-acetylglucosamine, is an important structural component in the cell walls of filamentous fungal that plays a critical role in fungi development and pathogenicity (Klis, 1994). The fluorochrome stain calcofluor binds preferentially to areas containing chitin and is widely used to determine cell wall integrity (Lewtak et al., 2014). In our study, a weaker and uneven fluorescence in the cell walls, particularly in the septa, was observed, which indicated that the chitin content in the cell walls 


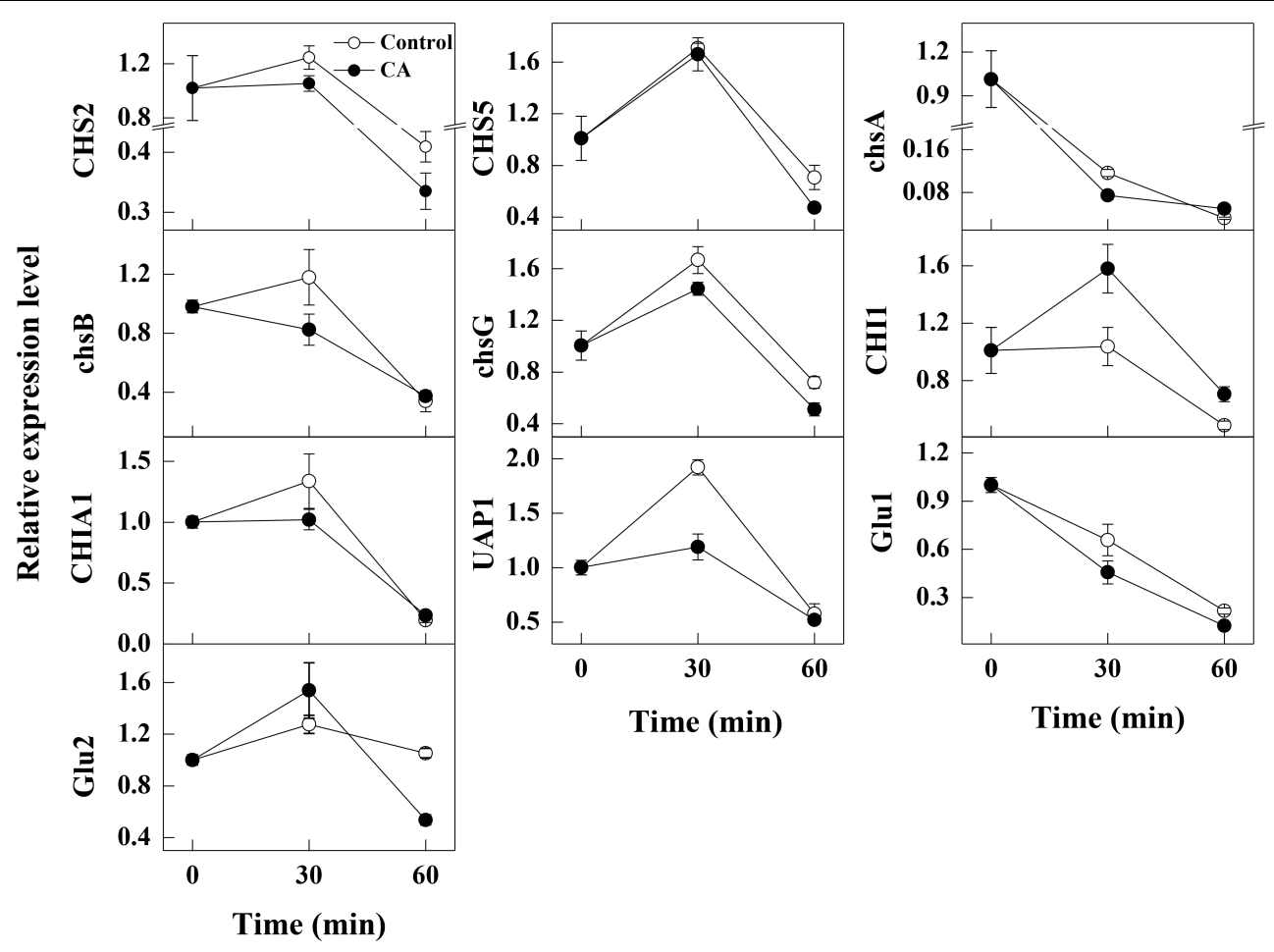

FIGURE $\mathbf{5}$ | Changes in the expression levels of the cell wall integrity related genes in control and 1/2MIC CA treated G. citri-aurantii mycelia. The data presented are the means of pooled data. Error bars indicate the SDs of the means $(n=3)$.

may be affected by CA treatment. This result was also confirmed by the chitin contents assay. These results demonstrated that the cell wall was an important antifungal target of CA.

In fungi, the chitin content is genetically regulated by some chitin synthase genes. Chitin synthases (CHS2, CHS5, chsA, chsB, and chsG) are located on the outer surface of the fungal mycelia and are responsible for the hyphal growth and conidiation (Mellado et al., 1996; Ichinomiya et al., 2002). Among them, CHS2 and chsA are closely related with cell wall integrity (Fujiwara et al., 2000; Soulie et al., 2003). In addition, CHS2 is responsible for the synthesis of septal chitin and serves indispensable roles in the formation of the primary septum (Fujiwara et al., 2000; Schmidt et al., 2002). CHS5 is required for maintaining the normal levels of chitin in S. cerevisiae, and mutants lacking CHS5 have a $75 \%$ reduction in cell wall chitin and altered morphology (Santos et al., 1997). As revealed in this study, the expression levels of the tested chitin synthases genes related to chitin synthesis were all repressed by CA treatment, suggesting that the build of cell wall might be destructed. This has been reported in previous studies that CA served as a cell wall target antifungal agent against $S$. cerevisiae cells by the inhibition of cell wall-synthesizing enzymes, such as $\beta$-(1,3)-glucan synthase and chitin synthase I (Bang et al., 2000; Shreaz et al., 2016). Meanwhile, the gene UAP1 responsible for producing the essential initial substrate (UDP-N-acetylglucosamine) in chitin biosynthesis, and its expression level was decreased after CA treatment, which indicated that the biosynthesis of UDP-N-acetylglucosamine and chitin might be impaired, as reported by another study (Maiti et al., 2015). Furthermore, the gene expression of CHII, a gene related to the hydrolysis of chitin, was stimulated, and this may accelerate the hydrolysis of chitin and therefore led to the breakdown of cell wall integrity (Takaya et al., 1998; Zhang et al., 2017). In our study, the increase of extracellular AKP activity and the decrease of chitin content also supported the hypothesis that CA inhibited the growth of G. citri-aurantii by affecting the formation of cell wall and the integrity of cell wall.

Meanwhile, glucans are also the components constituting the cell wall. Therefore, the structure and stability of fungal cell is also influenced by such cell wall degrading enzymes as Glu1 and Glu2 involved in glucan metabolism. These two enzymes are both $\beta$-1,3-glucan catabolic enzymes responsible for the hydrolysis of beta-D-glucose (Matsui et al., 2017). In our study, the expression levels of Glu1 and Glu2 were significantly lower than the control samples, indicating the metabolism of glucans might be greatly affected by the CA treatment. These results indicated that CA treatment not only inhibited chitin synthesis but also induced the hydrolysis of chitins, thereby affecting the formation of cell wall and destroying the cell integrity of G. citriaurantii.

\section{CONCLUSION}

In conclusion, our results showed that the impairment of cell wall caused by CA was prior to the damage of cell membrane, 
indicating that the main antifungal mechanism of CA against G. citri-aurantii is attributed to the impairment to the formation of cell wall and the disruption in cell wall integrity.

\section{AUTHOR CONTRIBUTIONS}

NT designed the research. QO performed the research. NT and QO analyzed the data. QO, XD, LL, and NT wrote the manuscript. All authors contributed to study design and provided

\section{REFERENCES}

Bang, K. H., Lee, D. W., Park, H. M., and Rhee, Y. H. (2000). Inhibition of fungal cell wall synthesizing enzymes by trans-cinnamaldehyde. Biosci. Biotech. Bioch. 64, 1061-1063. doi: 10.1271/bbb.64.1061

Duan, X. F., OuYang, Q. L., and Tao, N. G. (2018). Effect of applying cinnamaldehyde incorporated in wax on green mold decay in citrus fruits. J. Sci. Food. Agric. 98, 527-533. doi: 10.1002/jsfa.8490

Fujiwara, M., Ichinomiya, M., Motoyama, T., Horiuchi, H., Ohta, A., and Takagi, M. (2000). Evidence that the Aspergillus nidulans class I and class II chitin synthase genes, chsc and chsa, share critical roles in hyphal wall integrity and conidiophore development. J. Biochem. 127, 359-366. doi: 10. 1093/oxfordjournals.jbchem.a022616

Ghfir, B., Fonvieille, J. L., and Dargent, R. (1997). Influence of essential oil of Hyssopus officinalis on the chemical composition of the walls of Aspergillus fumigatus (Fresenius). Mycopathologia 138, 7-12. doi: 10.1023/A: 1006876018261

Hu, L., Wang, D., Liu, L., Chen, J., Xue, Y., and Shi, Z. (2013). Ca ${ }^{2+}$ efflux is involved in cinnamaldehyde-induced growth inhibition of Phytophthora capsici. PLoS One 8:e76264. doi: 10.1371/journal.pone.0076264

Ichinomiya, M., Motoyama, T., Fujiwara, M., Takagi, M., Horiuchi, H., and Ohta, A. (2002). Repression of chsB expression reveals the functional importance of class IV chitin synthase gene $c h s D$ in hyphal growth and conidiation of Aspergillus nidulans. Microbiology 148, 1335-1347. doi: 10.1099/ 00221287-148-5-1335

Jiang, X. (2015). Synthesis of Chitosan Schiff-base and its Antifungal Mechanism on Botrytis cinerea. Ph.D. thesis, Guiyang Medical University, Huaxi.

Klis, F. M. (1994). Cell wall assembly in yeast. Yeast 10, 851-869. doi: 10.1002/yea. 320100702

Leite, M. C. A., Bezerra, A. P. D. B., Sousa, J. P. D., Guerra, F. Q. S., and Lima, E. D. O. (2014). Evaluation of antifungal activity and mechanism of action of citral against Candida albicans. Evid Based Compl. Alt. 2014:378280. doi: 10.1155/2014/378280

Lewtak, K., Fiołka, M. J., Szczuka, E., Ptaszyńska, A. A., Kotowicz, N., Kołodziej, P., et al. (2014). Analysis of antifungal and anticancer effects of the extract from Pelargonium zonale. Micron 66, 69-79. doi: 10.1016/j.micron.2014. 06.001

Livak, K. J., and Schmittgen, T. D. (2001). Analysis of relative gene expression data using Real-Time quantitative PCR and the $2^{-\Delta \Delta C T}$ method. Methods 25, 402-408. doi: 10.1006/meth.2001.1262

Maiti, P., Ghorai, P., Ghosh, S., Kamthan, M., Tyagi, R. K., and Datta, A. (2015). Mapping of functional domains and characterization of the transcription factor Cph1 that mediate morphogenesis in Candida albicans. Fungal Genet. Biol. 83, 45-57. doi: 10.1016/j.fgb.2015.08.004

Matsui, M., Yokoyama, T., Nemoto, K., Kumagai, T., Terai, G., Tamano, K., et al. (2017). Identification of a putative FR901469 biosynthesis gene cluster in fungal sp. No. 11243 and enhancement of the productivity by overexpressing the transcription factor gene frbF. J. Biosci. Bioeng. 123, 147-153. doi: 10.1016/j. jbiosc.2016.08.007

McKay, A. H., Förster, H., and Adaskaveg, J. E. (2012). Efficacy and application strategies for propiconazole as a new postharvest fungicide for managing sour rot and green mold of citrus fruit. Plant Dis. 96, 235-242. doi: 10.1094/PDIS06-11-0525 input on the manuscript preparation, and given approval to the final version of the manuscript.

\section{FUNDING}

This study was supported by the National Natural Science Foundation of China (No. 31772364), Collaborative Innovation Center of New Chemical Technologies for Environmental Benignity and Efficient Resource Utilization, and Hunan Provincial Natural Science Foundation of China (2017JJ2247).

Mellado, E., Aufauvre-Brown, A., Gow, N. A., and Holden, D. W. (1996). The Aspergillus fumigatus chs $C$ and chs $G$ genes encode class III chitin synthases with different functions. Mol. Microbiol. 20, 667-679. doi: 10.1046/j.1365-2958.1996. 5571084.x

OuYang, Q. L., Tao, N. G., and Jing, G. X. (2016). Transcriptional profiling analysis of Penicillium digitatum, the causal agent of citrus green mold, unravels an inhibited ergosterol biosynthesis pathway in response to citral. BMC Genomics 17:599. doi: 10.1186/s12864-016-2943-4

OuYang, Q. L., Tao, N. G., and Zhang, M. L. (2018). A damaged oxidative phosphorylation mechanism is involved in the antifungal activity of citral against Penicillium digitatum. Front. Microbiol. 9:239. doi: 10.3389/fmicb.2018. 00239

Regnier, T., Combrinck, S., Veldman, W., and Du Plooy, W. (2014). Application of essential oils as multi-target fungicides for the control of Geotrichum citri-aurantii and other postharvest pathogens of citrus. Ind. Crop. Prod. 61, 151-159. doi: 10.1016/j.indcrop.2014.05.052

Santos, B., Duran, A., and Valdivieso, M. H. (1997). CHS5, a gene involved in chitin synthesis and mating in Saccharomyces cerevisiae. Mol. Cell. Biol. 17, 2485-2496. doi: 10.1128/MCB.17.5.2485

Schmidt, M., Bowers, B., Varma, A., Roh, D. H., and Cabib, E. (2002). In budding yeast, contraction of the actomyosin ring and formation of the primary septum at cytokinesis depend on each other. J. Cell Sci. 115, 293-302.

Shao, X., Cao, B., Xu, F., Xie, S., Yu, D., and Wang, H. (2015). Effect of postharvest application of chitosan combined with clove oil against citrus green mold. Postharvest Biol. Technol. 99, 37-43.

Shao, X., Cheng, S., Wang, H., Yu, D., and Mungai, C. (2013). The possible mechanism of antifungal action of tea tree oil on Botrytis cinerea. J. Appl. Microbiol. 114, 1642-1649. doi: 10.1111/jam.12193

Shreaz, S., Bhatia, R., Khan, N., Muralidhar, S., Basir, S. F., Manzoor, N., et al. (2011). Spice oil cinnamaldehyde exhibits potent anticandidal activity against fluconazole resistant clinical isolates. Fitoterapia 82, 1012-1020. doi: 10.1016/j. fitote.2011.06.004

Shreaz, S., Bhatia, R., Khan, N., Muralidhar, S., Manzoor, N., and Khan, L. A. (2013). Influences of cinnamic aldehydes on $\mathrm{H}^{+}$extrusion activity and ultrastructure of Candida. J. Med. Microbiol. 62, 232-240. doi: 10.1099/jmm. 0.036145-0

Shreaz, S., Wani, W. A., Behbehani, J. M., Raja, V., Irshad, M., Karched, M., et al. (2016). Cinnamaldehyde and its derivatives, a novel class of antifungal agents. Fitoterapia 2016, 116-131. doi: 10.1016/j.fitote.2016. 05.016

Smid, E. J., Koeken, J. G. P., and Gorris, L. G. M. (1996). "Fungicidal and fungistatic action of the secondary plant metabolites cinnarnaldehyde and carvone," in 'Modern Fungicides and Antifungal Compounds', eds H. Lyr, P. E. Russell, and H. D. Sisler (Andover: Intercept), 173-180.

Soulie, M. C., Piffeteau, A., Choquer, M., Boccara, M., and Vidal-Cros, A. (2003). Disruption of Botrytis cinerea class I chitin synthase gene Bcchs1 results in cell wall weakening and reduced virulence. Fungal Genet. Biol. 40, 38-46. doi: 10.1016/S1087-1845(03)00065-3

Takaya, N., Yamazaki, D., Horiuchi, H., Ohta, A., and Takagi, M. (1998). Cloning and characterization of a chitinase-encoding gene (chiA) from Aspergillus nidulans, disruption of which decreases germination frequency and hyphal growth. Biosci. Biotech. Biochem. 62, 60-65. doi: 10.1271/bbb. 62.60 
Talibi, I., Askarne, L., Boubaker, H., Boudyach, E. H., Msanda, F., Saadi, B., et al. (2012). Antifungal activity of Moroccan medicinal plants against citrus sour rot agent Geotrichum candidum. Lett. Appl. Microbiol. 55, 155-161. doi: 10.1111/j. 1472-765X.2012.03273.x

Tao, N. G., OuYang, Q. L., and Jia, L. (2014). Citral inhibits mycelial growth of Penicillium italicum by a membrane damage mechanism. Food Control 41, 116-121. doi: 10.1016/j.foodcont.2014.01.010

Wang, S. Y., Chen, P. F., and Chang, S. T. (2005). Antifungal activities of essential oils and their constituents from indigenous cinnamon (Cinnamomum osmophloeum) leaves against wood decay fungi. Bioresour. Technol. 96, 813-818. doi: 10.1016/j.biortech.2004.07.010

Wei, Y., Wei, Y., Xu, F., and Shao, X. (2018). The combined effects of tea tree oil and hot air treatment on the quality and sensory characteristics and decay of strawberry. Postharvest Biol. Technol. 136, 139-144. doi: 10.1016/j.postharvbio. 2017.11.008

Wu, Y. L., Duan, X. F., Jing, G. X., OuYang, Q. L., and Tao, N. G. (2017). Cinnamaldehyde inhibits the mycelial growth of Geotrichum citri-aurantii and induces defense responses against sour rot in citrus fruit. Postharvest Biol. Technol. 129, 23-28. doi: 10.1016/j.postharvbio.2017.03.004

Wuryatmo, E., Able, A. J., Ford, C. M., and Scott, E. S. (2014). Effect of volatile citral on the development of blue mould, green mould and sour rot on navel orange. Australas. Plant Pathol. 43, 403-411. doi: 10.1007/s13313-014-0281-z

Xie, X. M., Fang, J. R., and Xu, Y. (2004). Study of antifungal effect of cinnamaldehyde and citral on Aspergillus flavus. Food Sci. 25, 32-34. doi: 10. 3321/j.issn:1002-6630.2004.09.002

Xing, F. G., Hua, H. J., Selvaraj, J. N., Zhao, Y. J., Zhou, L., Liu, X., et al. (2014). Growth inhibition and morphological alterations of Fusarium verticillioides by cinnamon oil and cinnamaldehyde. Food Control 46, 343-350. doi: 10.1016/j. foodcont.2014.04.037

Yang, S. Z., Liu, L. M., Li, D. M., Xia, H., Su, X. J., Peng, L. T., et al. (2016). Use of active extracts of poplar buds against Penicillium italicum and possible modes of action. Food Chem. 196, 610-618. doi: 10.1016/j.foodchem.2015. 09.101

Zhang, S., Chen, C., Xie, T., and Ye, S. (2017). Identification and validation of reference genes for qRT-PCR studies of the obligate aphid pathogenic fungus Pandora neoaphidis during different developmental stages. PLoS One 12:e0179930. doi: 10.1371/journal.pone.0179930

Zhou, H. E., Tao, N. G., and Jia, L. (2014). Antifungal activity of citral, octanal and $\alpha$-terpineol against Geotrichum citri-aurantii. Food Control 37, 277-283. doi: 10.1016/j.foodcont.2013.09.057

Conflict of Interest Statement: The authors declare that the research was conducted in the absence of any commercial or financial relationships that could be construed as a potential conflict of interest.

The reviewer JH and handling Editor declared their shared affiliation.

Copyright (c) 2019 OuYang, Duan, Li and Tao. This is an open-access article distributed under the terms of the Creative Commons Attribution License (CC BY). The use, distribution or reproduction in other forums is permitted, provided the original author(s) and the copyright owner(s) are credited and that the original publication in this journal is cited, in accordance with accepted academic practice. No use, distribution or reproduction is permitted which does not comply with these terms. 